\title{
Morphology Control and Mechanical Properties of Liquid Crystalline Polymer-Polyamide Composite Fibers
}

\author{
Huimin WANG, Xiaoming TAO, Edward Newton, and Tai-Shung ChUnG* \\ Institute of Textiles and Clothing, The Hong Kong Polytechnic University, Hung Hom, Kowloon, Hong Kong, China \\ ${ }^{*}$ Institute of Materials Research and Engineering, 10 Kent Ridge Crescent, Singapore 119260, Singapore
}

(Received October 22, 2001; Accepted July 5, 2002)

\begin{abstract}
In this paper, we investigate the relationships between the viscosity ratio, morphology, and processing temperature of composite fibers comprising liquid crystalline polymer (LCP) and polyamide-66 (PA 66). Furthermore, we examine in details the effect of the LCP concentration on the phase microstructure and the mechanical properties of the composite fibers. The results show that, first, the optimal processing temperature of melt spinning of the composite fibers depends strongly on the viscosity ratio of LCP to PA 66 though the processing temperature range can be determined by the degradation temperature of PA 66 and the melting point of LCP. Secondly, the LCP phase structure/morphology in composite fibers can be controlled by the viscosity ratio of LCP to PA 66. Thirdly, the LCP phase structure/morphology are changed with LCP concentration for a fixed viscosity ratio/processing temperature. With an increase in LCP concentration, the morphology of LCP phase is changed from a fine fibril dispersed phase to a perfectly aligned continuous fiber reinforced phase in a rich LCP composite fiber. Finally, the mechanical properties of LCP composite fibers depend on the LCP phase structure/morphology and LCP concentration. The tensile properties of the composite fibers exhibit the positive synergy in the rich LCP composite fibers.

KEY WORDS Composite Fibers / Morphology Control / Viscosity Ratio / Processing Temperature / Liquid Crystalline Polymer (LCP) Concentration / Mechanical Properties /
\end{abstract}

The morphology of the materials, especially the morphology/microstructure of the LCP phase, has a strong influence on the mechanical properties of the composite materials comprising thermotropic liquid crystalline polymers (LCPs) and thermoplastic matrix. In general, the thermotropic LCPs do not directly transform to an isotropic melt when heated above their melting point, but to a state where the orientational long-range order is preserved whereas the long-range positional order breaks down. Thermotropic LCPs are essentially rigid-rod long-chain molecules with some irregularity or flexibility incorporated into the polymer chain to lower the melting point below the decomposition temperature. The rigid-rod molecular structure allows these materials to exhibit molecular order in a liquid mesophase. The highly oriented nature of LCPs produces highly anisotropic physical property and makes thermotropic LCPs quite attractive as a potential dispersed phase in in-situ reinforcing materials. ${ }^{1-22}$

The processing conditions affect the morphology and properties of the composites significantly. The processing conditions are also related to rheological properties of polymers, which determine the deformation and flow behavior of polymer materials in liquid, melt or solid form. In many industrial processes, polymer melts are subject to complex flows and temperature histories. To understand these complex flows, the response of the polymer melts in simple flow fields has been studied to determine material properties such as the viscosity, nor- mal stress coefficient and dynamic modulus. In turn, these material properties have been used to select the processing parameters for the polymers and to study the rheology-structure relationship of the polymers.

For composite systems of a liquid crystalline polymer (LCP), Vectra ${ }^{\mathrm{TM}} \mathrm{A}$, and polyamines (PA), ${ }^{2}$ for example, the viscosities of PA 66 and PA 6 decrease slowly with an increase of temperature, while the viscosity of Vectra A drops dramatically at $280^{\circ} \mathrm{C}$, but remains slightly changed above $295^{\circ} \mathrm{C}$. At constant frequency and above $295^{\circ} \mathrm{C}$, the mean value of the activation energy of Vectra ${ }^{\mathrm{TM}} \mathrm{A}$ is about $87.0 \mathrm{~kJ} \mathrm{~mol}^{-1}$, but jumps to a much higher value of about $407.0 \mathrm{~kJ} \mathrm{~mol}^{-1}$ if the melt temperature is below $295^{\circ} \mathrm{C}$. The activation energy of Vectra ${ }^{\mathrm{TM}} \mathrm{A}$ above $295^{\circ} \mathrm{C}$ is lower than PA 66 , which shows that the viscosity of PA 66 has larger temperature dependence than Vectra ${ }^{\mathrm{TM}} \mathrm{A}$. The viscosity of Vectra ${ }^{\mathrm{TM}} \mathrm{A}$ is less than that of PA 66 at temperatures higher than $290^{\circ} \mathrm{C}$, which indicates that Vectra ${ }^{\mathrm{TM}}$ A can form the fibrils in PA 66 matrix and reinforce PA 66 when blended together above this temperature.

The effect of processing conditions on the morphology and properties of the blends was predominantly investigated for injection molding, while more recently efforts were also put on making insitu composite fibers via melting spinning. ${ }^{1,10,23-28}$ The investigated systems mainly included $\mathrm{Vectra}^{\mathrm{TM}}$ A900e/polypropylen, ${ }^{23,24}$ LCP/poly(ether ether ketone)/poly(ether imide), ${ }^{25} \mathrm{LCP} /$ polycarbonate and 
polysulfone, ${ }^{26}$ LCP/PET, ${ }^{27-29}$ LCP/PES (polyether sulfone) $)^{11}$ and LCP/LCP. ${ }^{30}$ For example, the properties of polypropylene/Vectra ${ }^{\mathrm{TM}}$ A900 blend fibers with different weight ratios (100:2.5, 100:5, 100:10, 100:15) were investigated by Qin et al. ${ }^{23,24}$ They found that the single-stage drawing producing fibers had a slightly higher initial modulus than polypropylene, but a significantly lower tenacity.

For the composite fibers consisting of LCP and PES (polyether sulfone) as well as the blends of poly(phenyl-1,4-phenylene terephthalate) liquid crystalline polymer with polycarbonate and polysulfone, the tensile moduli and strengths of the composite fibers were consistent with the morphology of highly oriented LCP microfibrils with high aspect ratios dispersed in the thermoplastic fiber and the mechanical properties increased with increasing draw ratio. ${ }^{11,26}$ In addition, the fusion heat of the LCP phase in LCP/PES composite fibers increases continuously with draw down ratio, while the crystal-nematic transition temperature, $T_{\mathrm{m}}$, and glass transition temperature, $T_{\mathrm{g}}$, of the PES matrix are unaffected. ${ }^{11}$ The mechanical properties of the LCP/PET composite fibers could be well improved by heat treatment. ${ }^{28,29}$

Creveceur and Groeninckx ${ }^{25}$ evaluated the blend fibers of a thermotropic liquid crystalline polymer and a thermoplastic matrix of $75 \mathrm{wt} \%$ poly(ether ether ketone) and $25 \mathrm{wt} \%$ poly(ether imide) in terms of morphology and mechanical properties. The composite fibers containing more than $25 \mathrm{wt} \%$ LCP exhibited a morphology in which the LCP was dispersed as very long fibrils with good alignment of molecules in the spinning direction. The fibers followed the rule of mixtures behavior for stiffness $v s$. composition. For fiber containing 5 and $10 \mathrm{wt} \% \mathrm{LCP}$, both fibril aspect ratio and molecular orientation were lower, and the stiffness was below the rule of mixtures.

Most of the research on LCP/thermoplastics ${ }^{31-33}$ have been focused on the improvement of the mechanical properties of thermoplastics through the microfibrillar morphology of the LCP-dispersed phase formed in a suitable deformation history, in addition to the improvement of the melt processing of thermoplastics by adding a small amount of LCP to a conventional thermoplastic polymer. The LCPs can form the elongated fibrils under appropriate processing conditions, and reinforce the thermoplastic matrix. ${ }^{34-37}$ However, it has been found that the LCP domains do not always form fibrils for some LCP/thermoplastic composites. ${ }^{2,25-30}$ It is interesting that different processing conditions and different phase structure may result in a synergistic effect on the composite fibers. The objectives of this work are two folds. First, to investigate the relation- ship between the viscosity ratio and the morphology, and identify the optimal melt spinning temperature for the LCP-PA composite fibers. Second, to examine the effect of the LCP concentration on the LCP phase structure/morphology and the synergy of LCP composite fibers for a fixed processing temperature, and provide a guideline for the morphology control and property improvement of the composite fibers.

\section{EXPERIMENTS}

\section{Materials}

LCP Vectra ${ }^{\mathrm{TM}}$ A 950 used was a wholly aromatic copolyester consisting of $27 \mathrm{~mol} \%$ of 2,6hydroxynaphthoic acid (HNA) and $73 \mathrm{~mol} \%$ of $p$ hydroxybenzoic acid (HBA) produced by Hoechst Celanese Company. The polyamide-66 (PA 66) pellets employed were the product of Asahi Chemical Industry Co. Ltd., Japan. The LCP and PA 66 were dried in a vacuum oven at $120^{\circ} \mathrm{C}$ for $24 \mathrm{~h}$ prior to mixing. The blending was performed in a Brabender Plasticorder equipped with a mixing chamber of $50 \mathrm{~cm}^{3}$ and operated at $290^{\circ} \mathrm{C}$ and $75 \mathrm{rpm}$ for $5 \mathrm{~min}$. Weight ratios of LCP and PA 66 were 10/90; 20/80; 30/70; 40/60; 50/50, and 70/30, respectively. Fiber spinning was performed at $295^{\circ} \mathrm{C}$ by the melt extrusion of LCP, PA 66 and the LCP/PA 66 blends, followed by hot stretching the asspun fibers on a small scale drawing unit comprising of two pairs of advancing rollers and a hot plate. All fibers were spun from mono-filament die with $0.4 \mathrm{~mm}$ diameter and an aspect ratio of 2 . The drawing ratio is 4.

\section{DSC Experiments}

Thermal analysis was carried out using a PerkinElmer Pyris-1 DSC. The samples were heated from $100^{\circ} \mathrm{C}$ with the heating rate of $10^{\circ} \mathrm{C} \mathrm{min}^{-1}$. The temperature and heat flow of the instrument were carefully calibrated with highly pure indium and zinc prior to use.

\section{Thermogravimetric Analysis (TGA)}

The measurements of weight loss of LCP and PA 66 in air were performed on a Perkin-Elmer thermogravimetric analysis system TGA 7 with $5^{\circ} \mathrm{C} \min ^{-1}$.

\section{Rheological Experiment}

The rheological experiments were performed using Advanced Rheometric Expansion System (modelARES) with parallel plates produced by Rheometric Scientific, Inc. The diameter of parallel plates was $25 \mathrm{~mm}$. The frequency was set at $62.8 \mathrm{rad} \mathrm{s}^{-1}$ and the applied strain was $50 \%$. The plate gap was $1.5 \mathrm{~mm}$. The 
viscosity ratio was determined by the viscosity of LCP to PA 66 at the same test conditions.

\section{Polarized Light Microscopy (PLM)}

The phase structure/morphology of the LCP blends and composite fibers under polarized light was observed with an Olympus BX50 microscope equipped with a Linkam TP93 hot stage with a maximum heating and cooling rate of $90^{\circ} \mathrm{C} \mathrm{min}-1$. The sample was first sandwiched between two glass cover slips on a hot plat. The micrographs were taken at a temperature of $270^{\circ} \mathrm{C}$ where the PA 66 phase was molten and LCP fibrils were below their melting point. Magnification used was $200 \mathrm{X}$.

\section{Scanning Electron Microscopy (SEM)}

The morphology of the fracture surfaces of the composite fibers was observed in a scanning electron microscope (SEM, Philips-XL 30). All fractured surfaces were coated with a thin layer of gold prior to SEM examination.

\section{Mechanical Properties}

The tests of the mechanical properties were carried out on an Instron tensile tester with a gauge length of $50 \mathrm{~mm}$ and an extension rate of $10 \mathrm{~mm} \mathrm{~min}^{-1}$. The test was performed at $25^{\circ} \mathrm{C}$ and $65 \%$ relative humidity. The diameter of composite fibers is about $0.2 \mathrm{~mm}$. The effective numbers are not less than 5 and the standard deviation is $<10 \%$.

\section{RESULTS AND DISCUSSION}

\section{Determination of Optimal Processing Temperature}

Temperature is one of the important processing parameters. The processing temperature of a single polymer is more than its melting temperature and below its degradation temperature, while the processing temperature range of two conventional polymer blends is from the melting temperature of higher melting temperature polymer to the degradation temperature of lower degradation temperature polymer. The DSC and TGA curves of Vectra ${ }^{\mathrm{TM}}$ A 950 and PA 66 are shown in Figures 1 and 2, respectively. It can be seen that the melting temperatures ( $T_{\mathrm{m}}$, peak temperature) of Vectra ${ }^{\mathrm{TM}} \mathrm{A}$ 950 and PA 66 are 280 and $263^{\circ} \mathrm{C}$, respectively. The TGA experimental result (Figure 2) exhibits that the Vectra $^{\mathrm{TM}}$ A 950 and PA 66 start slightly degrading at 450 and $360^{\circ} \mathrm{C}$, though their degradation temperatures $\left(T_{\mathrm{d}}\right)$ are 480 and $400^{\circ} \mathrm{C}$, respectively. This indicates that the processing temperature range of Vectra ${ }^{\mathrm{TM}} \mathrm{A}$ 950 and PA 66 composite system should be in 280 to $360^{\circ} \mathrm{C}$.

For LCP in-situ composites, the viscosity ratio of

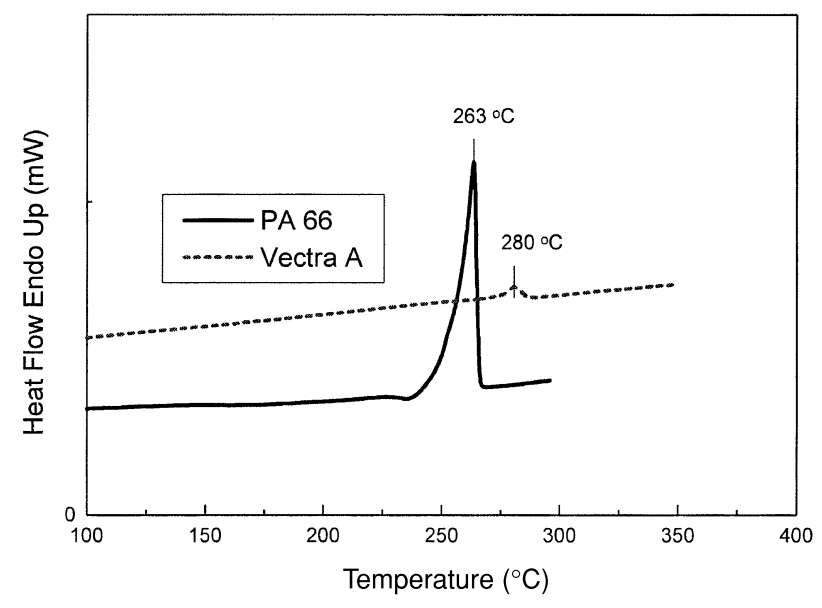

Figure 1. DSC curves of PA 66 and LCP.

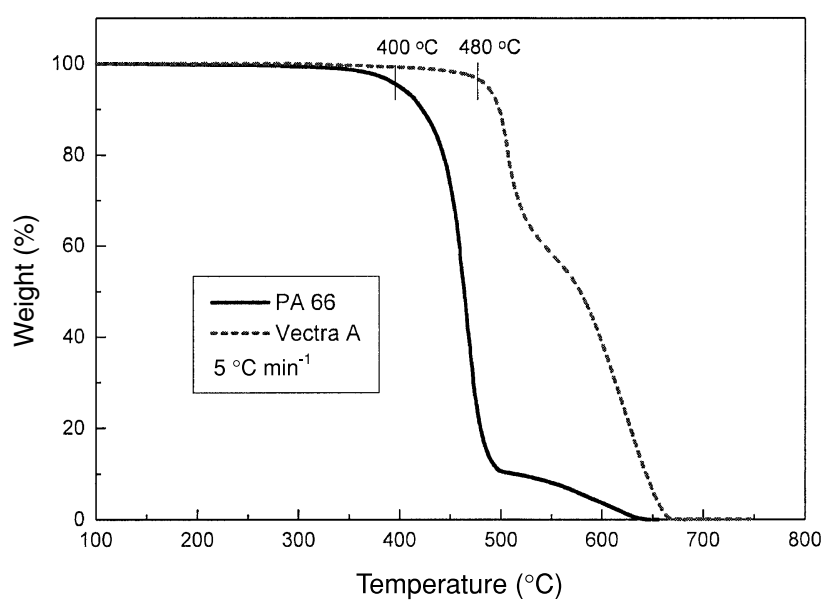

Figure 2. TGA curves of PA 66 and LCP.

LCP to matrix has strong influence on their morphological and mechanical properties, and is a critical factor in determining the composite morphology. For good fibrillation to be achieved, the viscosity of the dispersed LCP phase should be lower than that of the matrix $^{2,14,37}$ (i.e., $\eta_{\mathrm{LCP}} / \eta_{\text {Matrix }}<1$ ). Temperature dependence curves of viscosity ratio of LCP to PA 66 at $62.8 \mathrm{rad} \mathrm{s}^{-1}$ are shown in Figure 3. It is very clear that the viscosity ratio of LCP to PA 66 is more than 1 below $290^{\circ} \mathrm{C}$ and is less than 1 above $290^{\circ} \mathrm{C}$. For example, the viscosity ratio of LCP to PA 66 is 3.42 at $285^{\circ} \mathrm{C}$, while the viscosity ratio of LCP to PA 66 is 0.75 at $295^{\circ} \mathrm{C}$. The corresponded morphology of the dispersed LCP phase is shown in Figure 4. The optical micrographs show that good fibrillation can be achieved when the viscosity ratio of the dispersed LCP phase to the PA 66 matrix is 0.75 (less than 1 ) at $295^{\circ} \mathrm{C}$. The dispersed LCP domains tend to be spherical or cluster-like when the viscosity ratio of the dispersed LCP phase to the PA 66 matrix is 3.42 (more than 1 ) at $285^{\circ} \mathrm{C}$. This shows that the LCP/PA 66 composite systems can form a better reinforcing phase at a processing temperature of $295^{\circ} \mathrm{C}$ than at a processing temperature of $285^{\circ} \mathrm{C}$. 


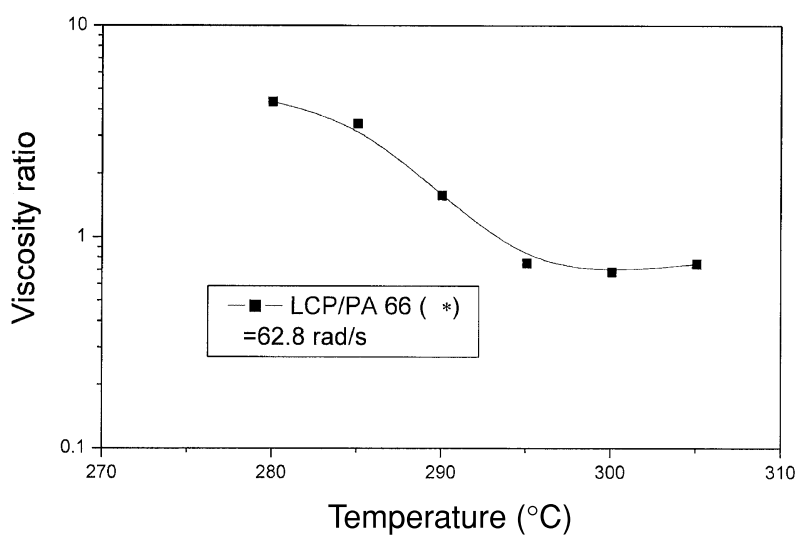

Figure 3. Temperature dependence curves of viscosity ratio of LCP to PA 66 at a frequencies of $62.8 \mathrm{rad} \mathrm{s}^{-1}$.

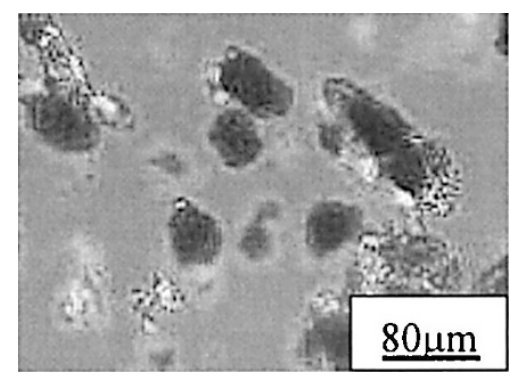

A: a viscosity ratio of 3.42 at $285^{\circ} \mathrm{C}$

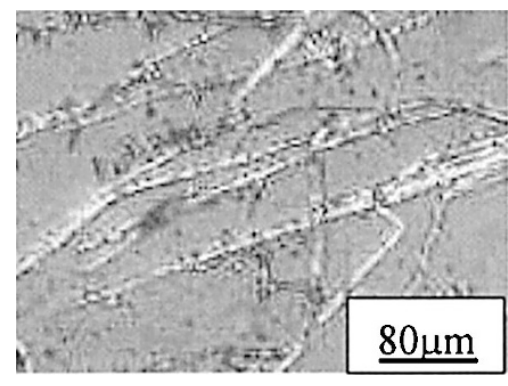

B: a viscosity ratio of 0.75 at $295^{\circ} \mathrm{C}$

Figure 4. Optical micrographs of LCP/PA 66 (20/80) composite system at different viscosity ratios.

The processing temperature can change the viscosity ratio of LCP composite systems for a fixed LCP concentration, which results in different LCP reinforcing phase structure/morphology in LCP composites. ${ }^{1,14,37}$ In fact, the good fibrillation can only be achieved when the viscosity of the dispersed LCP phase is less than that of the PA 66 matrix above $290^{\circ} \mathrm{C}$. That is to say, LCP is able to form the fibrils in PA 66 matrix and reinforce PA 66 when the processing temperature is above $290^{\circ} \mathrm{C}$, but it is difficult to form LCP fibrils in PA 66 matrix below $290^{\circ} \mathrm{C}$. However, the high processing temperature will easily result in the degradation of PA 66. In general, the processing temperature of PA 66 should be below $300^{\circ} \mathrm{C}$. Based on the ratio of LCP to PA 66 and the instability of PA 66 at high processing temperature, we think that $295^{\circ} \mathrm{C}$ is an optimal processing temperature for melt spinning of LCP/PA 66 com- posite fiber.

Effect of LCP Concentration on Morphology of LCP/PA Composite Fibers

The processing conditions, mixing equipment and LCP concentration have a crucial effect on the morphology of immiscible polymer-polymer composite systems. Flow type also plays a decisive role in the LCP deformation. Shear flow tends to break up the polydomain structure, while extensional flow may consolidate and orient it. ${ }^{39}$ In the present work, the transformation of the LCP into fibrils may take place in two stages. First, there is an elongational flow at the spinneret hole entrance where deformation can take place. Secondly, there exists an elongational strain below the spinneret, which can deform the LCP phase.

Figures $5 \mathrm{a}$ and $5 \mathrm{~b}$ are the hot-stage optical micrographs of composite fibers with LCP/PA 66 (wt/wt) ratios of 10/90 and 20/80 at a processing temperature of $295^{\circ} \mathrm{C}$. It can be seen that the LCP and PA 66 exist in separate phases. The LCP fibrils are fine and chaotically dispersed in PA matrix. The hot-stage optical micrograph of composite fibers with LCP/PA 66 (wt/wt) ratios of 30/70 is shown in Figure 5c. The short LCP fibrils are mostly aligned in the direction of flow, however, the aspect ratio (L/D) ratio of the fibrils is very small. The hot-stage optical micrographs of composite fibers with LCP/PA $66(\mathrm{wt} / \mathrm{wt})$ ratios of $40 / 60$ and $50 / 50$ are illustrated in Figures 5d and 5e. As LCP concentrations are increased, the most fibrils are aligned in PA matrix in the direction of flow. Figure $5 \mathrm{f}$ displays the hot-stage optical micrograph of LCP/PA 66 (70/30) composite fibers. The LCP forms the continuous fibril phases with increasing the concentrations which are perfectly aligned in the direction of flow.

Compared Figure 5b with Figure 4B, it can be found that different processing conditions can result in different phase structure/morphology of LCP/PA 66 composites even if they have the same level of LCP concentrations. For LCP/PA 66 blends (Figure 4B), the LCP phase dispersed in PA 66 matrix forms long fibrils due to the shear stress of screw in the mixer and the diameter of the fibrils is from 10 to $50 \mu \mathrm{m}$. For LCP/PA 66 composite fibers (Figure $5 \mathrm{~b}$ ), a good dispersion and an elongational flow at the spinneret hole entrance can result in LCP fine fibrous structure and the diameter of fine fibrils is below $10 \mu \mathrm{m}$. This is due to the fact that, during fiber spinning, the LCP particles dispersed in the matrix through elongation into cylindrical shapes break up into smaller droplets in quiescent regions. These drops are elongated again in high-shear regions, break up again, and so on until viscous stress equilibrates the interfacial stress. ${ }^{39}$ At the spinneret hole entrance, a 


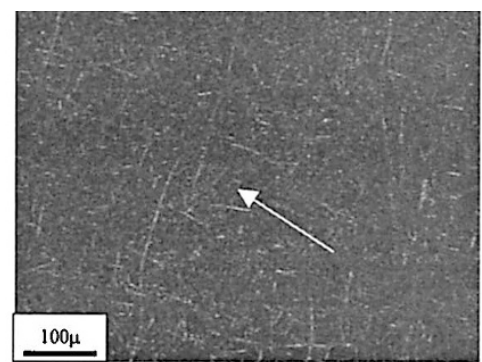

a: $(10 / 90)$

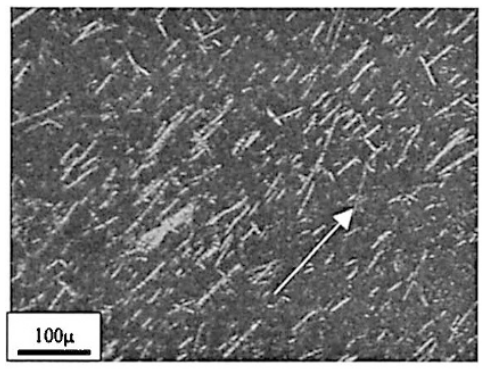

c: $(30 / 70)$

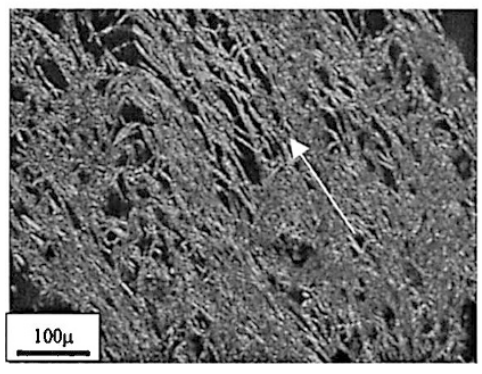

e: $(50 / 50)$

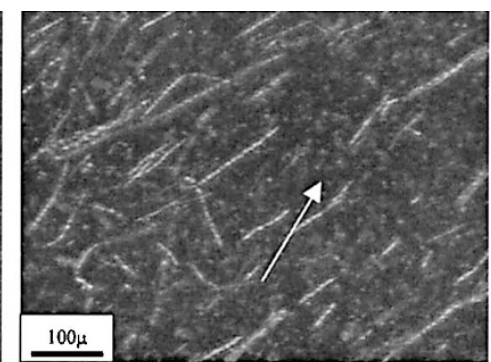

b: $(20 / 80)$

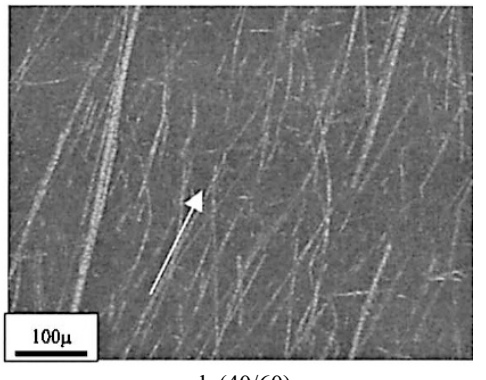

$\mathrm{d}:(40 / 60)$

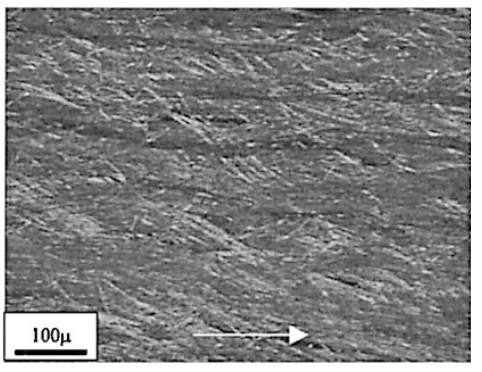

f: $(70 / 30)$

Figure 5. Optical micrographs of LCP/PA 66 composite fibers (Direction of arrow is direction of flow).

rapid acceleration flow begins, which produces an elongational flow that deforms fine fibril form. In addition, the LCP fibrous morphology is stable since the activation energy of LCP phase is very high below $280^{\circ} \mathrm{C} .^{2}$

We carried out the SEM observations of the transverse cross-fractured morphology of the LCP/PA 66 $(10 / 90,20 / 80,30 / 70,50 / 50$, and 70/30) composite fibers at a processing temperature of $295^{\circ} \mathrm{C}$. The SEM photographs are shown in Figure 6. It is obvious that PA 66 is immiscible with LCP, and there are two distinct phases in the composite fibers. The morphology of LCP phase changes with the LCP concentration. LCP exhibits a fine fibril dispersed phase in the PA 66 matrix if the LCP concentration is low. With an increase in LCP concentration, the morphology of LCP phase varies from a fine fibril dispersed phase to a perfectly aligned continuous fibrils reinforced phase in the rich LCP composition samples. Thus, the aspect ratio (L/D) of the LCP phase increases with increasing LCP concentration. The above observation is in agreement with LCP fibrils in other matrices well documented in the literature. ${ }^{9,40,41}$ In short, the formation mechanisms of LCP fibrils in in-situ composite fibers are (A) the LCP component of the composite systems forms an elongated, fibrous phase that orients in the direction of flow when an extensional component is present during processing of the melt. Because of the long relaxation times of the LCP, the fibrous nature of the dispersed phase may be preserved even in cases where the extensional flow is followed by a simple shear flow. (B) a rapid acceleration flow begins, which produces an elongational flow that deforms dispersed phase into fibril form, at the spinneret hole entrance.

\section{Effect of Morphology on Mechanical Properties of LCP/PA 66 Composite Fibers}

In general, the mechanical properties of LCP insitu composite systems do not follow the rule of mixture ${ }^{27,39}$ and often exhibit the negative or positive synergy, ${ }^{25}$ which is related to the processing technology, fibril orientation and LCP concentration, etc. ${ }^{9}, 40,41$ The tensile strengths of LCP/PA 66 composite fibers with different LCP concentration are shown in Figure 7, while the dependence of tensile modulus on LCP concentration in LCP/PA 66 composite fibers is displayed in Figure 8. The tensile moduli increase with 


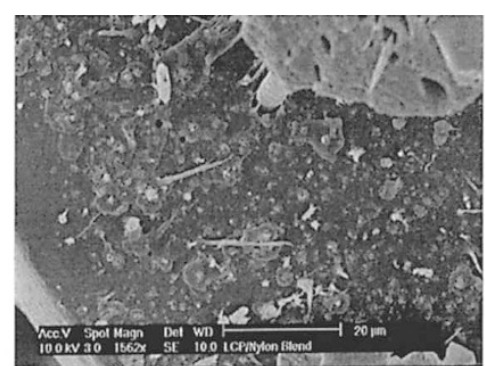

a: $(10 / 90)$

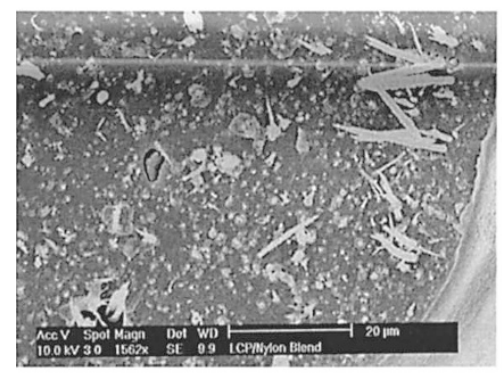

c: $(30 / 70)$

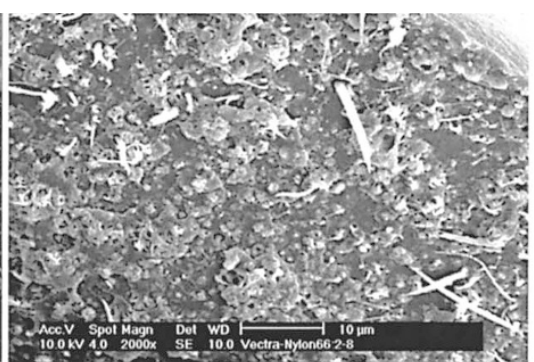

b: $(20 / 80)$

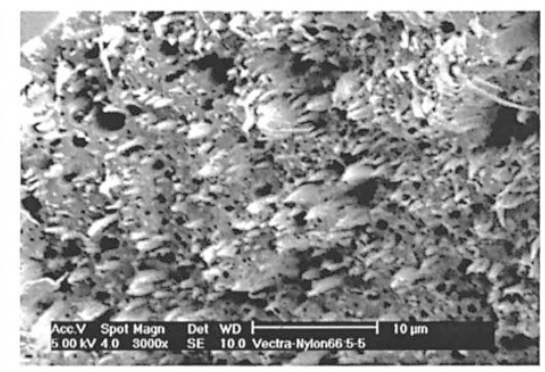

d: $(50 / 50)$

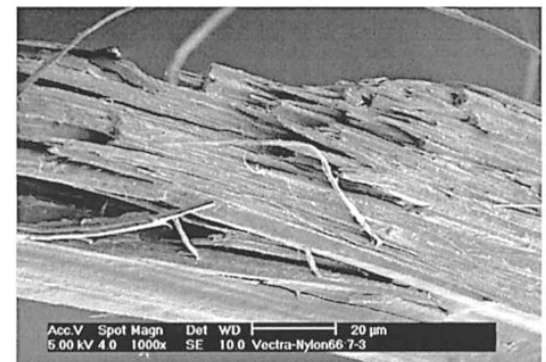

e: $(70 / 30)$

Figure 6. SEM photographs of fractured surface of LCP/PA 66 composite fibers.

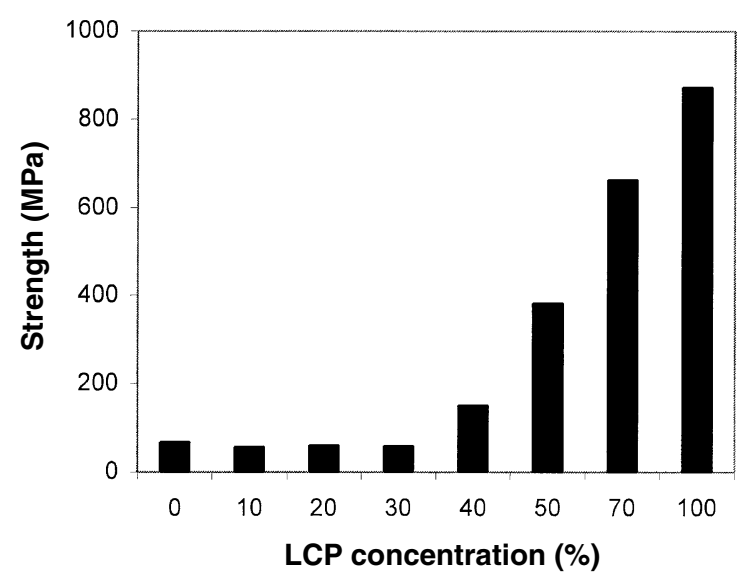

Figure 7. Dependence of tensile strength on LCP concentration in LCP/PA 66 composite fibers.

an increase in LCP concentration, especially in the rich LCP concentration. For example, the tensile moduli of PA and the composite fibers with LCP/PA 66 (wt/wt) ratios of 10/90, 20/80, and 30/70 are 585, 717, 1098, and $1619 \mathrm{MPa}$, respectively. But the tensile modulus of the composite fibers is sharply enhanced at $40 \mathrm{wt} \%$ LCP concentration and has the positive synergistic ef-

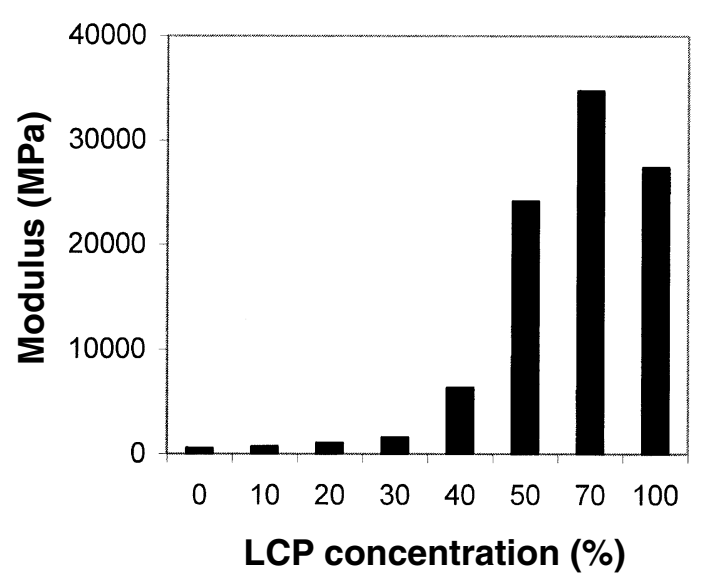

Figure 8. Dependence of tensile modulus on LCP concentration in LCP/PA 66 composite fibers.

fect if the LCP concentration is rich, indicating that the LCP phase can easily orient and form the continuous fibril phase. The tensile strengths increase with an increase in LCP concentration only when LCP concentration is above $40 \mathrm{wt} \%$. This indicates that the LCP phase has the reinforcing effect when the LCP concentration is greater than $40 \mathrm{wt} \%$. Compared to the pure 
PA 66 fiber, it can be found that the $40 \mathrm{wt} \% \mathrm{LCP}$ composite fiber shows a $982 \%$ increase in tensile modulus and a $123 \%$ increase in tensile strength. The mechanical properties of LCP/PA 66 composite fibers depend mainly on their morphology. Although the long fibrils can be observed in low LCP concentration like LCP/PA $66(10 / 90$ and 20/80) composite fibers, the LCP concentration is too low to reinforce PA 66 matrix and the tensile strengths of the composite fibers are mainly controlled by the properties of matrix. In the low LCP concentration, based on mechanics of composite materials, the tensile strength can be approximately shown as: $\sigma_{\mathrm{c}} \approx V_{\mathrm{m}} \sigma_{\mathrm{m}}$. Here $\sigma_{\mathrm{c}}$ is the strength of composite materials, while $V_{\mathrm{m}}$ and $\sigma_{\mathrm{m}}$ are the volume fraction and the strength of matrix, respectively. The tensile strength of the composite fiber is lower than that of matrix and exhibits the negative synergistic effect since $V_{\mathrm{m}}$ is below 1. For example, the tensile strength of pure PA is $67.8 \mathrm{MPa}$, while the tensile strengths of composite fibers with LCP/PA 66 (wt/wt) ratios of 10/90, 20/80, and $30 / 70$ are $56.9,58.9$, and $60.7 \mathrm{MPa}$, respectively.

In addition, from the hot-stage optical micrographs of composite fibers with LCP/PA 66 (wt/wt) ratios of $10 / 90$ and 20/80, it can be known that another reason of their tensile strengths lower than pure PA 66 fiber's one is that the fine LCP fibrils chaotically dispersed in PA66 matrix. For LCP/PA (30/70) composite fiber, the unremarkable reinforcing effect is because the $\mathrm{L} / \mathrm{D}$ ratio of the LCP fibrils is too small though the most short LCP fibrils can be aligned in the direction of flow. Thereafter, the most LCP fibrils are aligned in PA matrix in the direction of flow and produce the remarkable reinforcing effect, as the LCP concentrations are increased. The composite fibers containing more than $40 \mathrm{wt} \%$ LCP exhibited a morphology in which the LCP was dispersed as very long fibrils with good alignment of molecules in the flowing direction (Figures $5 \mathrm{~d}, 5 \mathrm{e}$, and $5 \mathrm{f}$ ). The LCP forms the continuous fibril phases with increasing further the concentrations which are perfectly aligned in the direction of flow and the reinforcing effect is more remarkable. Our experimental results on LCP/PA 66 system are similar to LCP/PEI system, ${ }^{39}$ as well as the blend fibers of a LCP and a thermoplastic matrix of $75 \mathrm{wt} \%$ PEEK and $25 \mathrm{wt} \%$ PEI reported by Creveceur and Groeninckx. ${ }^{25}$ Their experimental results showed that the composite fibers containing more than $25 \mathrm{wt} \%$ LCP exhibited a morphology in which the LCP was dispersed as very long fibrils with good alignment of molecules in the spinning direction.

In addition, it is found that the synergistic effect of the tensile modulus is much better than that of the tensile strength. This is because the tensile modulus depends on the structure and orientation of macro-

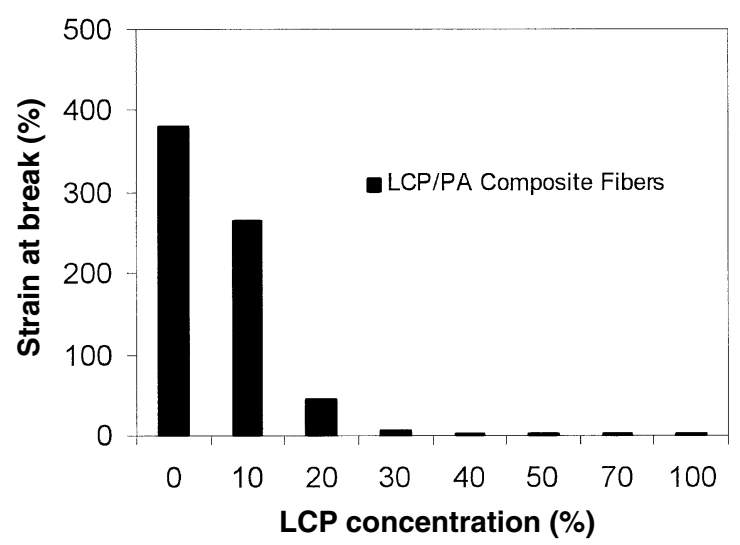

Figure 9. Dependence of strain at break on LCP concentration in LCP/PA 66 composite fibers.

molecules, while the strength is related to the bond energy. For composite system, the function of the reinforcing phase is to bear the load, while the main function of the matrix is to transfer the stress.

In the mechanical property tests, it is interesting to note that the pure PA fiber and the composite fibers containing below $30 \mathrm{wt} \% \mathrm{LCP}$ show clear yield points and ductile fractures, while the samples containing equal and more than $30 \mathrm{wt} \%$ LCP do not yield and give brittle fractures. The dependence of strain at break on LCP concentration in LCP/PA fibers is shown in Figure 9. The strains at break decrease with increasing LCP concentration, but remain invariant above $30 \mathrm{wt} \%$ LCP concentration.

For LCP composite systems, the mechanical properties of composite fibers are controlled by the LCP phase structure/morphology, while the LCP phase structure/morphology is related to LCP concentration and processing conditions, such as processing temperature (viscosity ratio) and processing method (flow types of melt). The viscosity ratio is a critical factor in determining the blend morphology. The good fibrillation can be achieved when the viscosity of the dispersed LCP phase is less than that of the PA 66 matrix above $290^{\circ} \mathrm{C}$. In addition, an elongational flow at the spinneret hole entrance makes both of LCP and PA 66 orient in the direction of flow. Since the orientation relaxation time of LCP is longer than the stress relaxation time, ${ }^{42}$ thus the orientation LCPs achieved during processing is frozen in the solid state more easily than for flexible chain PA 66. LCP phase exists in PA matrix in the form of fibrils. Furthermore, in low LCP concentration, the disorientation or shrinkage of PA 66 take place since the relaxation time is related to the activation energy, $\Delta E, \tau \propto e^{\Delta \mathrm{E} / \mathrm{RT}}$, and the activation energies of LCP and $\mathrm{PA}$ are $407.0 \mathrm{~kJ} \mathrm{~mol}^{-1}$ and $109.6 \mathrm{~kJ} \mathrm{~mol}^{-1}$, 2 respectively, so the relaxation time of PA 66 is shorter than $\mathrm{LCP}$ relaxation time. The disorientation or shrinkage 
of PA 66 results in LCP fibrils not to align in the direction of flow, but to chaotically disperse in PA66 matrix. LCP fibrils lost their reinforcement effect. The mechanical properties of composite fibers are controlled by the properties of matrix. Moreover, in the rich LCP concentration, the LCP fibrils can form the continuous reinforcing phase and the composite fibers exhibit the excellent mechanical properties. On the other hand, if the LCP content is too high ( $>80 \%$ ), the LCP fibrils in the matrix would tend to coalesce into a sheet-like structure, resulting in poor reinforcement. ${ }^{25}$ The tensile modulus of the composite fibers has a positive synergy, as it is higher than the value predicted by the mixture law in the rich LCP concentration, especially around $70 \mathrm{wt} \%$.

\section{CONCLUSION}

The viscosity ratio of LCP to PA 66 varies with temperature and is less than 1 above $290^{\circ} \mathrm{C}$. Good fibrillation can be achieved when the viscosity ratio of the dispersed LCP phase to the PA 66 matrix is 0.75 (less than 1) at $295^{\circ} \mathrm{C}$. The dispersed LCP domains tend to be spherical or cluster-like when the viscosity ratio of the dispersed LCP phase to the PA 66 matrix is 3.42 (more than 1) at $285^{\circ} \mathrm{C}$. The morphology of the LCP composite fibers can be controlled by adjusting LCP concentration, in addition to viscosity ratio, processing conditions and methods. The morphology of the LCP phase changes with the LCP concentration for a fixed processing condition. With an increase in the LCP concentration, the morphology of LCP phase varies from a fine fibril dispersed phase to a perfectly aligned continuous fibrils reinforced phase if the LCP concentration is high. Good mechanical properties of composite fibers can be achieved with high LCP concentration due to a perfectly aligned continuous fibrils reinforced phase. The tensile modulus of the composite fibers has a positive synergy as it is higher than the value predicted by the mixture law in the rich LCP concentration, especially around $70 \mathrm{wt} \%$. The investigation of the relationships between the processing technology, morphology and mechanical properties can provide a guideline for the design, research and development of new polymer composite fibers as well as the investigation of nanopolymer composite fibers we are currying out.

\section{REFERENCES}

1. H. M. Wang, K. W. Lee, T. S. Chung, and M. Jaffe, Polym. Compos., 21, 114 (2000).

2. H. M. Wang, K. W. Lee, and T. S. Chung, Polym. Adv. Technol., 11, 153 (2000).
3. T. M. Malik, P. J. Carreau, and N. Chapleau, Polym. Eng. Sci., 29, 600 (1990).

4. A. Mehta and A. I. Isayev, Polym. Eng. Sci., 31, 971 (1991).

5. S. C. Tjong, S. L. Liu, and R. K. Y. Li, J. Mater. Sci., 30, 353 (1995).

6. S. C. Tjong, S. L. Liu, and R. K. Y. Li, J. Mater. Sci, 31, 479 (1996).

7. H. M. Wang and G. Hinrichsen, Int. Polym. Process., 11, 26 (1997).

8. S. G. James, A. M. Donald, and W. A. Macdonald, Mol. Cryst. Liq. Cryst., 153, 491 (1987).

9. A. Siegmann, A. Dagan, and S. Kenig, Polymer, 26, 1325 (1985).

10. T. S. Chung, Polym. Eng. Sci., 26, 901 (1986).

11. H. M. Wang, X. S. Yi, and G. Hinrichsen, Polym. J., 29, 881 (1997).

12. G. Kiss, Polym. Eng. Sci., 27, 410 (1987).

13. J. P. D. Souza and D. G. Baird, Polymer, 37, 1985 (1996).

14. D. Beery, S. Kenig, and A. Siegmann, Polym. Eng. Sci., 31, 459 (1991).

15. G. Sawhney, S. K. Gupta, and A. Misra, J. Appl. Polym. Sci., 62, 1395 (1996).

16. T. S. Chung, G. W. Calundann, and A. J. East, "Handbook of Polymer Science and Technology", by N. P. Cheremisinoff, Ed., New York, N.Y., 1989, p 625.

17. S. H. Kim, S. W. Park, and E. S. Gil, J. Appl. Polym. Sci., 67, 1383 (1998).

18. S. Lee, S. M. Hong, Y. Seo., T. S. Park, S. S. Hwang, and K. U. Kim, Polymer, 35, 519 (1994).

19. K. Cho, T. Kong, and D. Lee, Polym. J., 29, 904 (1997).

20. M. Kozlowski, and F. P. La Mantia, J. Appl. Polym. Sci., 66, 969 (1997).

21. A. G. C. Machiels, J. V. Dam, A. P. D. Boer, and B. Norder, Polym. Eng. Sci., 37, 1512 (1997).

22. R. E. Jerman and D. G. Baird, J. Rheol., 25, 272 (1981).

23. Y. Qin, D. L. Brydon, R. R. Mather, and R. H. Wardman, Polymer, 34, 1196 (1993).

24. Y. Qin, D. L. Brydon, R. R. Mather, and R. H. Wardman, Polymer, 34, 1203 (1993).

25. G. Creveceur and G. Groeninckx, Polym. Compos., 13, 244 (1992).

26. M. N. B. Skovby, J. Kops, and R. A. Weiss, Polym. Eng. Sci., 31, 954 (1991).

27. B. Y. Shin and I. J. Chung, Polym. Eng. Sci., 30, 13 (1990).

28. A. K. Mithal, A. Tayebi, and C. H. Lin, Polym. Eng. Sci., 31, 1533 (1991).

29. B. Liang, L. Pan, and X. He, J. Appl. Polym. Sci., 66, 217 (1997).

30. W. C. Lee and A. T. Di Benedtto, Polym. Eng. Sci., 32, 400 (1992).

31. K. G. Blizard and D. G. Baird, Polym. Eng. Sci., 27, 653 (1987).

32. F. P. La Mantia, A. Valenza, M. Paci, and P. L. Maganini, Polym. Eng. Sci., 30, 7 (1990).

33. J. Meissner, Macromolecular Symposia, 56, 25 (1992).

34. B. R. Bassett and A. F. Yee, Polym. Compos., 11, 10 (1990).

35. D. E. Turek and G. P. Simon, Polymer, 34, 2750 (1993).

36. J. P. D. Souza and D. G. Baird, Polymer, 37, 1985 (1996).

37. K. Min, J. L. White, and J. F. Fellers, Polym. Eng. Sci., 24 
1327 (1984)

38. K. F. Wissbrun, G. Kiss, and F. N. Cogswell, Chem. Eng. Commun., 53, 194 (1987).

39. Y. Seo, "Handbook of Engineering Polymeric Materials", N. P. Cheremisinoff, Ed., Marcel Dekker, Inc., New York, N.Y., 1997, p 585.
40. R. A. Weiss, W. Huh, and L. Nicolais, Polym. Eng. Sci., 27, 684 (1987).

41. D. R. Paul and C. B. Bucknall, Ed., "Polymer Blends", Academic Press, New York, N.Y., 2000, p 429.

42. R. E. Jerman and D. G. Baird, J. Rheol., 25, 272 (1981). 